\title{
Factors Affecting Compliance to a Gluten-Free Diet in Pediatric Populations with Celiac Disease
}

\author{
Vítor Macedo-Campos ${ }^{1,2,3,4, \S}$ Rui Macedo-Campos ${ }^{1,2,3,4, \S} \quad$ Filipa Pinto-Ribeiro ${ }^{1,3,4}$ \\ Henedina Antunes $1,2,3,4$
}

1 School of Medicine, University of Minho, Braga, Portugal

${ }^{2}$ Gastroenterology, Hepatology and Nutrition Unit, Pediatric Department and Clinical Academic Center, Hospital de Braga, Braga, Portugal

${ }^{3}$ Life and Health Sciences Research Institute (ICVS), School of

Medicine, University of Minho, Braga, Portugal

${ }^{4}$ ICVS/3B's-PT Government Associate Laboratory, Braga-Guimarães, Portugal

J Child Sci 2021;11:e1-e13.
Address for correspondence Vítor Manuel Macedo-Campos, Rua das Portelas, N342, Góios Barcelos 4755-248, Portugal

(e-mail: vitormaccampos@hotmail.com).
Abstract
Keywords
- celiac disease
- gluten-free diet
- adherence
- pediatric
- systematic review

Celiac disease (CD) is a multisystemic autoimmune disorder triggered by gluten, and the only known remedy available for this malady is a gluten-free diet (GFD). Therefore, we performed a systematic review to correlate the influence of different factors in compliance to a GFD. We searched PubMed database, from inception to April 2019. As inclusion criteria we considered population under 18 years, confirmed diagnosis of CD without related comorbidities and the study objective being the factors affecting compliance to a GFD. The variables compared were age, parent's education level, parental knowledge about $C D$, family type, celiac association membership, quality of life, and perception of difficulties in maintaining a GFD. We identified 1,414 articles, 35 articles were eligible for full text assessment and 12 were included in the study since they studied similar variables. Our work has found some limitations namely a variety of methods to assess GFD compliance, a limiting definition of compliance, a parental bias in data, an absence of standardization in age categories, and a majority of studies being observational in their nature. Age as well as parental knowledge of $C D$ and family type are key factors in pediatric GFD compliance. Nevertheless environmental, social, and family factors were also related with compliance. Further studies are needed to fully disclose the causality relation between these factors and compliance.

\section{Introduction}

The prevalence of celiac disease (CD) in Europe is approximately $1 \%{ }^{1}$ The pathophysiology for $\mathrm{CD}$ involves a multisystemic autoimmune-mediated disorder to gluten, a protein most commonly found in wheat, rye, and barley resulting in injury to the small bowel mucosa. ${ }^{2}$ Genetic susceptibility with HLA DQ-2 and/or DQ-8 positivity is strongly associated with the disease. ${ }^{2}$

$\bar{\S}$ These authors contributed equally to this work.

received

September 19, 2020

accepted after revision

November 18, 2020
DOI https://doi.org/

10.1055/s-0040-1722218.

ISSN 2474-5871.
Diagnosing $C D$ is challenging due to its nonspecific and heterogeneous clinical presentation. The symptoms can vary in intensity but commonly it presents with abdominal symptoms such as malabsorption, discomfort, loose stools, and flatulence ${ }^{3}$ and a variety of nonintestinal symptoms that include short stature, infertility, delayed puberty, anemia, liver abnormalities, joint and muscular disorders, neurological complications, psychiatric disorders and cutaneous and mucosal manifestations. ${ }^{4}$ Importantly, CD can also affect asymptomatic patients. ${ }^{3}$ (c) 2021. The Author(s).

This is an open access article published by Thieme under the terms of the Creative Commons Attribution License, permitting unrestricted use, distribution, and reproduction so long as the original work is properly cited. (https://creativecommons.org/licenses/by/4.0/)

Georg Thieme Verlag KG, Rüdigerstraße 14, 70469 Stuttgart, Germany 
e2 Factors Affecting Compliance to a Gluten-Free Diet Macedo-Campos et al.

Regarding the treatment of $\mathrm{CD}$, the only knowledgeable efficient treatment is a gluten-free diet (GFD). ${ }^{3}$ Without the exposure to gluten, the symptoms as well as the damage inflicted, regress and the patient becomes asymptomatic. ${ }^{2}$ Nevertheless, it represents a major lifelong change in lifestyle. Compliance with GFD can be challenging, onerous, expensive, and imposes difficulties to the patient. ${ }^{5}$ Hence, the need for a systematic review to identify factors that interfere with GFD compliance and to recognize predictors of noncompliance as well as modifiable factors that positively influence compliance is fully justified.

\section{Methods}

\section{Search Strategy}

We searched the PubMed database for literature regarding the compliance to GFD in pediatric CD from inception to April 16, 2019. The terms used to perform the search were as follows: celiac or celiac or gluten sensitive enteropathy AND diet\$ or nutrition\$ or GFD or gluten-free or gluten free AND advice or adherence or compliance or concordance or prescription or intervention or management AND child\$ or pediatric\$. No filter was applied.

\section{Eligibility Criteria for Studies and Participants}

The inclusion criteria were a study population that included parents of or children under 18 years old throughout the entire study course; confirmed diagnostic of CD without related comorbidities; and focus on the factors influencing compliance to a GFD.

The exclusion criteria were articles not written in English and gray literature.

\section{Study Selection and Data Extraction}

Study selection was performed independently by two authors (V.M.-C. and R.M.-C.). Discrepancies were resolved through discussion among them or by consulting a third author (H.A.). A PRISMA flowchart (-Fig. 1) was used to perform this record.

Prior to data extraction, the authors through the analysis of the papers herein included develop an excel form to

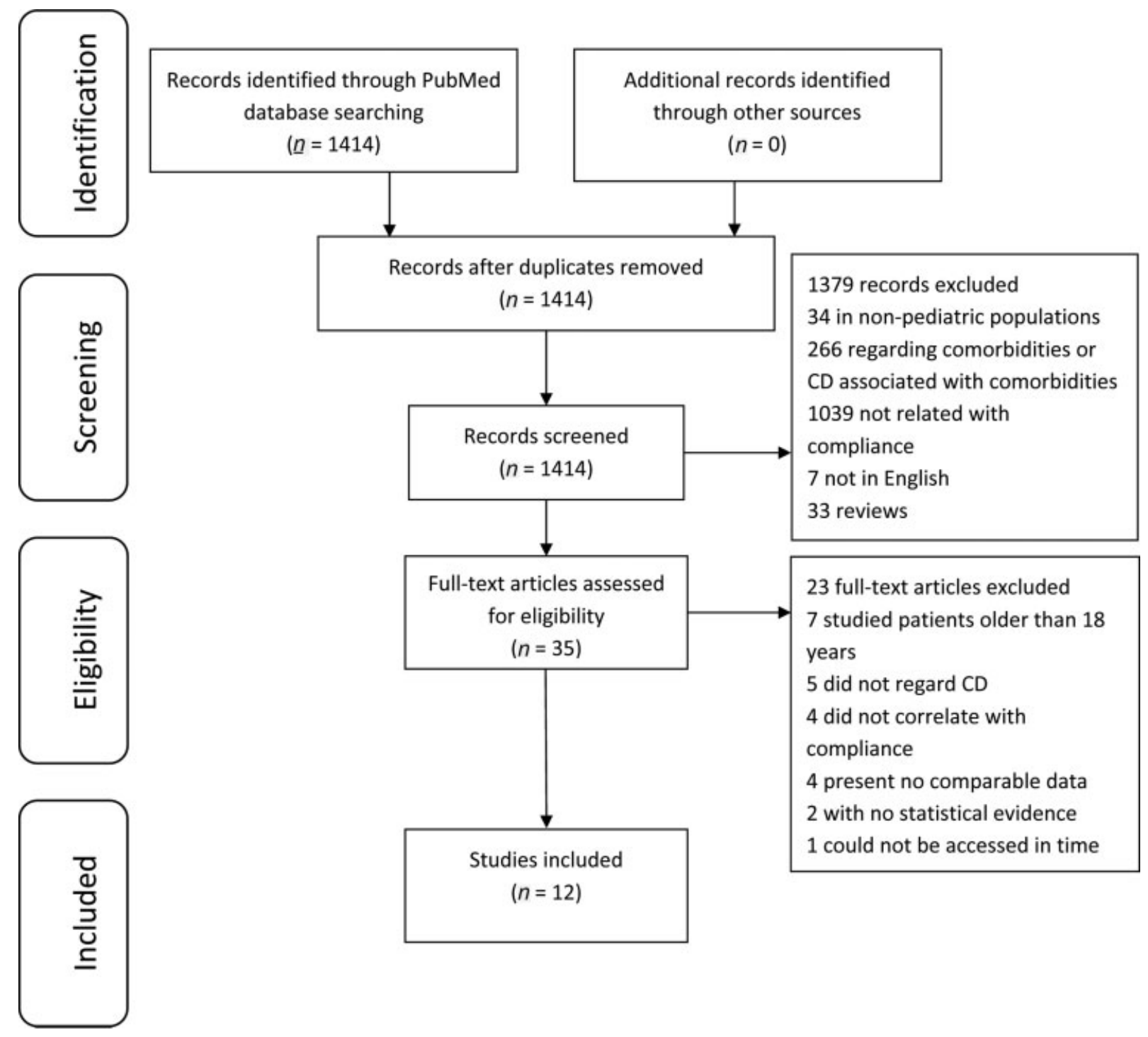

Fig. 1 Flowchart representing the study selection process. 
Table 1 Quality assessment of case-control studies

\begin{tabular}{|l|l|l|l|l|l|l|l|l|l|l|l|l|l|}
\hline & $\mathbf{1}$ & $\mathbf{2}$ & $\mathbf{3}$ & $\mathbf{4}$ & $\mathbf{5}$ & $\mathbf{6}$ & $\mathbf{7}$ & $\mathbf{8}$ & $\mathbf{9}$ & $\mathbf{1 0}$ & $\mathbf{1 1}$ & $\mathbf{1 2}$ & \\
\hline Chauhan et al & + & + & - & - & + & + & $?$ & - & + & + & + & $?$ & Good \\
\hline Wagner et al & + & + & - & - & + & + & $?$ & - & + & + & + & - & Medium \\
\hline Ljungman and Myrdal et al & + & - & - & + & - & + & $?$ & - & + & - & + & - & Poor \\
\hline
\end{tabular}

([-], “No"; [?], “Cannot Determine/Not Applicable/Not Reported”; [+], "Yes") (1. Was the research question or objective in this paper clearly stated and appropriate?; 2 . Was the study population clearly specified and defined?; 3 . Did the authors include a sample size justification?; 4 . Were controls selected or recruited from the same or similar population that gave rise to the cases (including the same timeframe)?; 5 . Were the definitions, inclusion and exclusion criteria, algorithms or processes used to identify or select cases and controls valid, reliable, and implemented consistently across all study participants?; 6 . Were the cases clearly defined and differentiated from controls?; 7 . If less than $100 \%$ of eligible cases and/or controls were selected for the study, were the cases and/or controls randomly selected from those eligible?; 8. Was there use of concurrent controls?; 9 . Were the investigators able to confirm that the exposure/risk occurred prior to the development of the condition or event that defined a participant as a case?; 10. Were the measures of exposure/risk clearly defined, valid, reliable, and implemented consistently (including the same time period) across all study participants?; 11 . Were the assessors of exposure/risk blinded to the case or control status of participants?; 12 . Were key potential confounding variables measured and adjusted statistically in the analyses? If matching was used, did the investigators account for matching during study analysis?)

systematically extract data (V.M.-C. and R.M.-C.). The form contemplated the following detailed parameters: title, authors, country of origin, year of publication, study design, participant number, mean participant age, the specific aim of study, methods used to assess compliance, and factors related with GFD compliance.

\section{Study Quality and Assessment of Risk Bias}

Quality was individually assessed by two authors (V.M.-C. and R.M.-C.). The authors resolved any disagreement through discussion of each dissonant parameter. When agreement could not be reached, a third author was consulted (H.A.).

Since the articles retrieved were cohort, cross-sectional, and case-control studies, the authors decided to assess quality using the National Heart, Lung and Blood Institute Study Quality
Assessment Tools. ${ }^{6}$ This checklist comprises 12 questions for case-control studies and 14 questions for cross-sectional and cohort studies. The questions must be answered using "Yes," "No," or "Cannot be applied/Not answered/Not reported."

After finalizing the quality assessment, the articles were divided into three groups rated as "Good," "Medium," or "Poor" according to their final scores. To obtain these subcategories, we excluded the questions in which all the articles scored the same. This information is summarized in - Tables $\mathbf{1}$ and $\mathbf{2}$.

\section{Results}

\section{Description of Study Selection}

The flowchart in - Fig. 1 shows a schematic presentation of the selection process of studies included. ${ }^{7}$ After searching

Table 2 Quality assessment of cross-sectional studies

\begin{tabular}{|l|l|l|l|l|l|l|l|l|l|l|l|l|l|l|l|}
\hline & $\mathbf{1}$ & $\mathbf{2}$ & $\mathbf{3}$ & $\mathbf{4}$ & $\mathbf{5}$ & $\mathbf{6}$ & $\mathbf{7}$ & $\mathbf{8}$ & $\mathbf{9}$ & $\mathbf{1 0}$ & $\mathbf{1 1}$ & $\mathbf{1 2}$ & $\mathbf{1 3}$ & $\mathbf{1 4}$ & \\
\hline Mager et al & + & + & + & + & - & - & - & + & + & - & + & $?$ & $?$ & + & Good \\
\hline Anson et al & + & + & + & + & - & - & - & + & - & - & + & + & $?$ & - & Medium \\
\hline Sarkhy et al & + & + & $?$ & + & + & - & - & + & + & - & + & + & $?$ & + & Good \\
\hline Charalampopoulos et al & + & + & + & + & - & - & - & - & + & - & + & + & $?$ & + & Medium \\
\hline Taghdir et al & + & + & + & + & - & - & - & + & + & - & + & $?$ & $?$ & - & Medium \\
\hline Khurana et al & + & - & $?$ & + & - & - & - & + & + & + & + & $?$ & $?$ & - & Poor \\
\hline MacCulloch and Rashid & + & + & + & + & + & - & - & + & + & - & + & + & $?$ & - & Good \\
\hline Garg and Gupta & + & + & $?$ & + & - & - & - & + & + & - & + & + & $?$ & + & Good \\
\hline Roma et al & + & + & $?$ & + & - & - & - & - & - & - & + & + & $?$ & - & Poor \\
\hline
\end{tabular}

([-], “No"; [?], “Cannot determine/not applicable/not reported”; [+], “Yes”) (1. Was the research question or objective in this paper clearly stated?; 2. Was the study population clearly specified and defined?; 3 . Was the participation rate of eligible persons at least $50 \%$ ?; 4 . Were all the subjects selected or recruited from the same or similar populations (including the same time period)? Were inclusion and exclusion criteria for being in the study prespecified and applied uniformly to all participants?; 5 . Was a sample size justification, power description, or variance and effect estimates provided?; 6. For the analyses in this paper, were the exposure(s) of interest measured prior to the outcome(s) being measured?; 7 . Was the timeframe sufficient so that one could reasonably expect to see an association between exposure and outcome if it existed?; 8 . For exposures that can vary in amount or level, did the study examine different levels of the exposure as related to the outcome (e.g., categories of exposure, or exposure measured as continuous variable)?; 9. Were the exposure measures (independent variables) clearly defined, valid, reliable, and implemented consistently across all study participants?; 10. Was the exposure(s) assessed more than once over time?; 11. Were the outcome measures (dependent variables) clearly defined, valid, reliable, and implemented consistently across all study participants?; 12 . Were the outcome assessors blinded to the exposure status of participants?; 13 . Was loss to follow-up after baseline $20 \%$ or less?; 14 . Were key potential confounding variables measured and adjusted statistically for their impact on the relationship between exposure(s) and outcome(s)?). 
e4 Factors Affecting Compliance to a Gluten-Free Diet Macedo-Campos et al.

PubMed using the keywords as described earlier, we obtained a total of 1,414 results. Nevertheless, 1,379 papers were excluded based on their title or/and abstract. Exclusion was based on the article type (systematic review, metaanalyses, comment, expert opinion, or letter), CD being associated with some comorbidity, compliance to GFD not being the main aim, and population over 18 years old. Of the 35 articles that remained for full text assessment, 23 articles were not eligible based on the following premises: seven studied population with patients older than 18 years old, five did not regard $\mathrm{CD}$, four did not correlate with compliance, four presented no comparable data, two with no statistical evidence and one could not be accessed in time for this review. A total of 12 articles were finally analyzed.

\section{Methodological Quality}

Of the included studies, nine are cross-sectional (75\%) and three are case-control (25\%) studies. Two cross-sectional studies and one case-control article were rated as "Poor." This rating was tied to the following assessments: Khurana et $\mathrm{al}^{8}$ lacked information regarding population details and Roma et $\mathrm{al}^{9}$ and Ljungman and Myrdal ${ }^{10}$ did not discriminate the factors affecting compliance, namely those that were evaluated as a dichotomic variable when more discrimination was possible.

The cross-sectional papers that scored the lowest were because of the following reasons: justifying and pretending the results of statistical analysis, exposure being measured before assessing the outcome, the time frame used, number of exposure assessment, number of participants lost to follow-up (not applicable to this type of study), and identification of potential confounding factors.
Regarding case-control studies, the items where all papers scored the lowest were the justification for the sample size and the use of concurrent controls.

\section{Characterization of the Population}

Data concerning the population at study is summarized in - Table 3. A population of 1,579 children was included in our study. None of the papers used populations smaller than 40 patients? Four articles ${ }^{10-13}$ did not display the mean age and two described it using a median statistic. ${ }^{14,15}$ Therefore, we were able to estimate an average $C D$ patient age of 10.3 years. The papers report to different geographic areas with five being from Europe, ${ }^{9,10,12,14}$ three from India, $, 11,13$ another three from Middle East,, ${ }^{5,16,17}$ and two from Canada. ${ }^{15,18}$ Except for two of them, ${ }^{10,17}$ all papers were published within the last decade. - Table 3 also elucidates on the method and criteria used for the diagnosis of $C D$.

\section{Characterization of the Study}

The analyses of $\mathbf{-}$ Tables $\mathbf{4}$ and $\mathbf{5}$ summarize the results of each paper considering the following categories: demographic factor, household, child related, parent related, dietary related, disease related, personality related, and quality of life (QoL).

\section{Adhesion Assessment}

Adhesion was measured heterogeneously between studies. One study $^{8}(9 \%)$ evaluated compliance measuring antitissue transglutaminase antibodies (t-TG) levels whereas 11 studies ${ }^{5,9-18}$ (91\%) relied on self-reported information with only one verifying it through measurement of t-TG levels. ${ }^{18}$ Self-reported compliance can be divided in questionnaires ${ }^{5,9,10,12-16,18}$ or

Table 3 Characterization of the population at study and criteria for the diagnosis of CD

\begin{tabular}{|c|c|c|c|c|c|c|c|}
\hline Authors & Country & Year & Study design & $\begin{array}{l}\text { Study } \\
\text { population }\end{array}$ & Age mean \pm SD & $\begin{array}{l}\text { Criteria used for } \\
\text { the diagnosis } \\
\text { of } C D\end{array}$ & $\begin{array}{l}\text { Biopsy to } \\
\text { confirm CD }\end{array}$ \\
\hline Mager et al & Canada & 2018 & Cross sectional & 372 & $\begin{array}{l}10.4 \pm 3.8(\mathrm{CD}) \\
10.9 \pm 4.0 \\
\text { (controls) }\end{array}$ & Nondisclosed & Performed \\
\hline Chauhan et al & India & 2010 & Case-control & 70 & - & ESPGHAN 2012 & Performed \\
\hline Anson et al & Israel & 1989 & Cross sectional & 43 & 10.7 & Nondisclosed & Performed \\
\hline Sarkhy et al & Saudi Arabia & 2015 & Cross sectional & 133 & 9.9 & Nondisclosed & $\begin{array}{l}\text { Performed in } 94 \% \\
\text { of population }\end{array}$ \\
\hline Wagner et al & Austria & 2016 & Case-control & 376 & - & Nondisclosed & Performed \\
\hline $\begin{array}{l}\text { Ljungman } \\
\text { and Myrdal }\end{array}$ & Sweden & 1993 & Case-control & 47 & - & ESPGHAN 1969 & Performed \\
\hline Taghdir et al & Iran & 2016 & Cross sectional & 65 & $11.3 \pm 3.8$ & $\begin{array}{l}\text { ESPGHAN criteria } \\
\text { (year nondisclosed) }\end{array}$ & Performed \\
\hline $\begin{array}{l}\text { Charalampopoulos } \\
\text { et al }\end{array}$ & Greece & 2013 & Cross sectional & 90 & 12.1 (median) & ESPGHAN 2012 & Performed \\
\hline Roma et al & Greece & 2010 & Cross sectional & 73 & 10.4 & ESPGHAN 1990 & Performed \\
\hline Khurana et al & India & 2014 & Cross sectional & 50 & 9.06 & ESPGHAN 1990 & Performed \\
\hline $\begin{array}{l}\text { MacCulloch } \\
\text { and Rashid }\end{array}$ & Canada & 2014 & Cross Sectional & 126 & 12 (median) & Nondisclosed & Performed \\
\hline Garg and Gupta & India & 2014 & Cohort & 134 & - & ESPGHAN 1990 & Performed \\
\hline
\end{tabular}

Abbreviations: -, value was not disclosed; CD, celiac disease; ESPGHAN, European Society for Paediatric Gastroenterology Hepatology and Nutrition. 


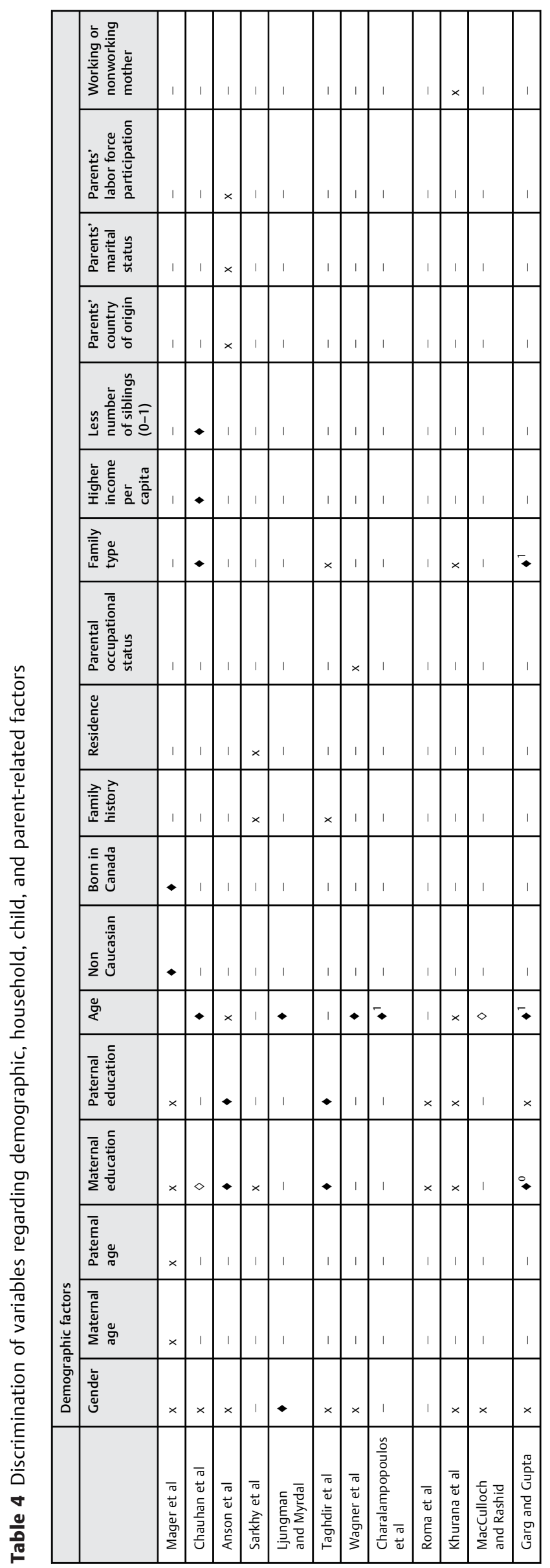

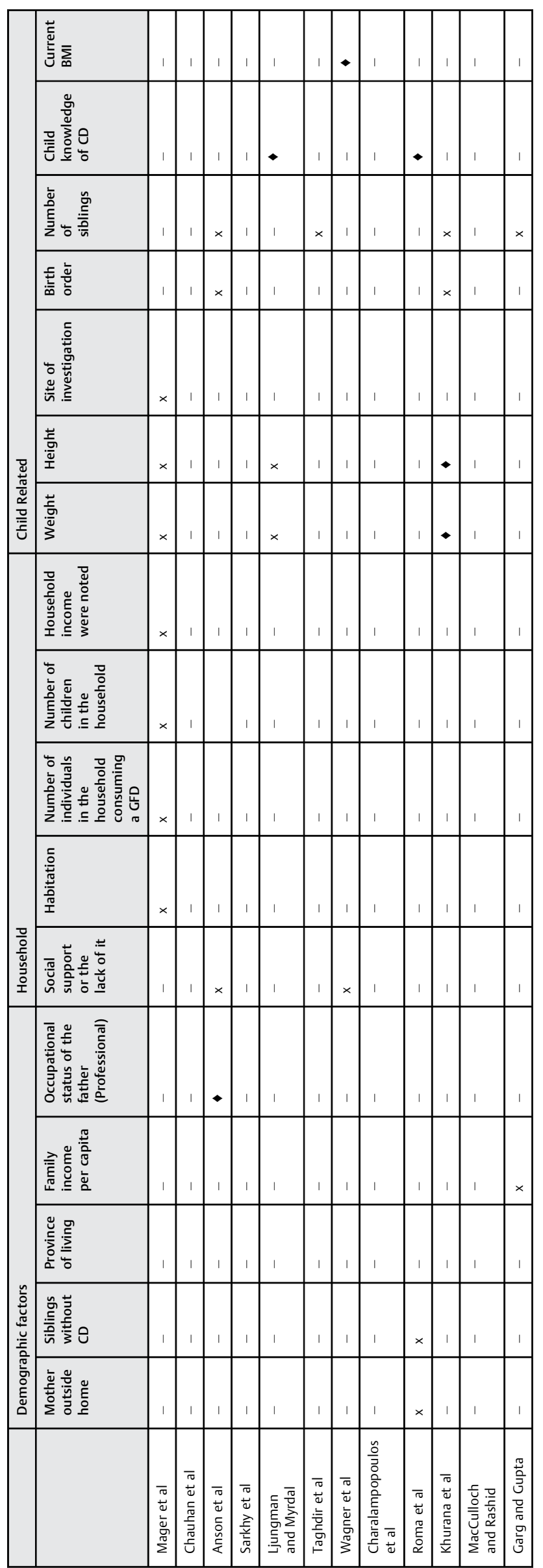




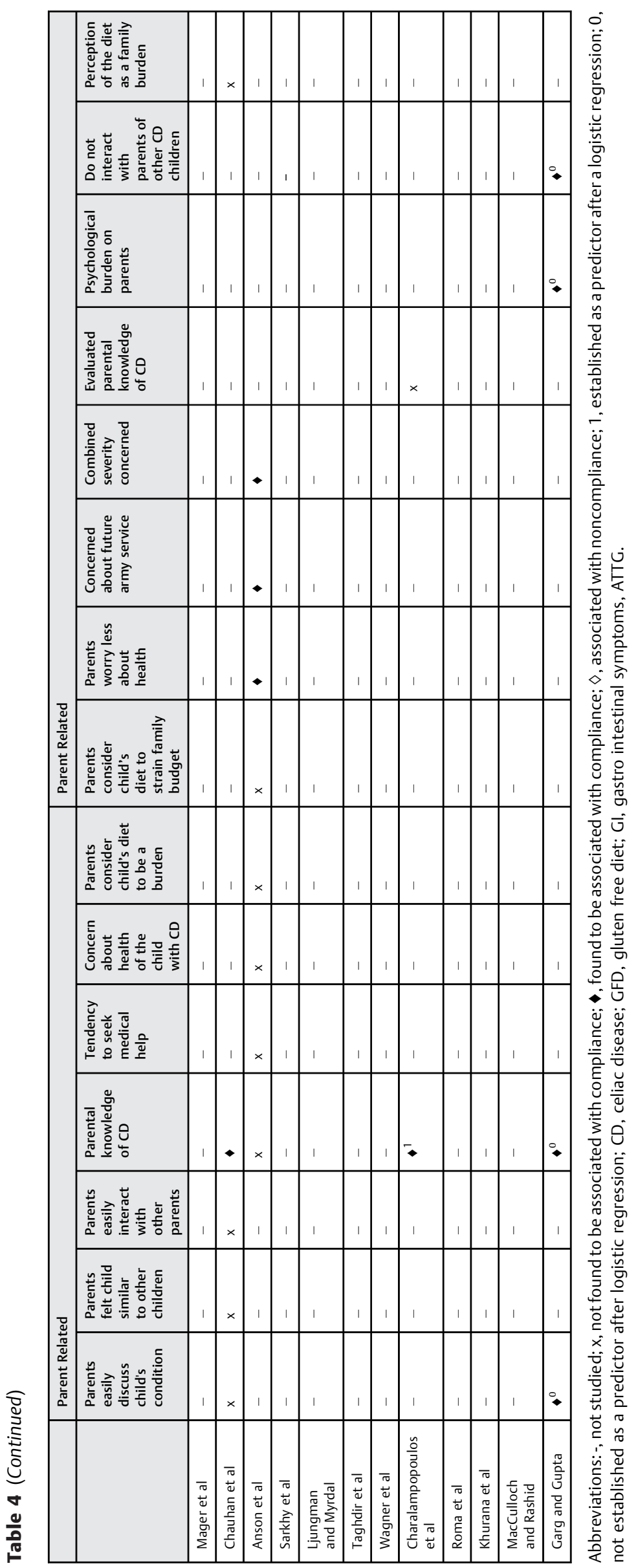




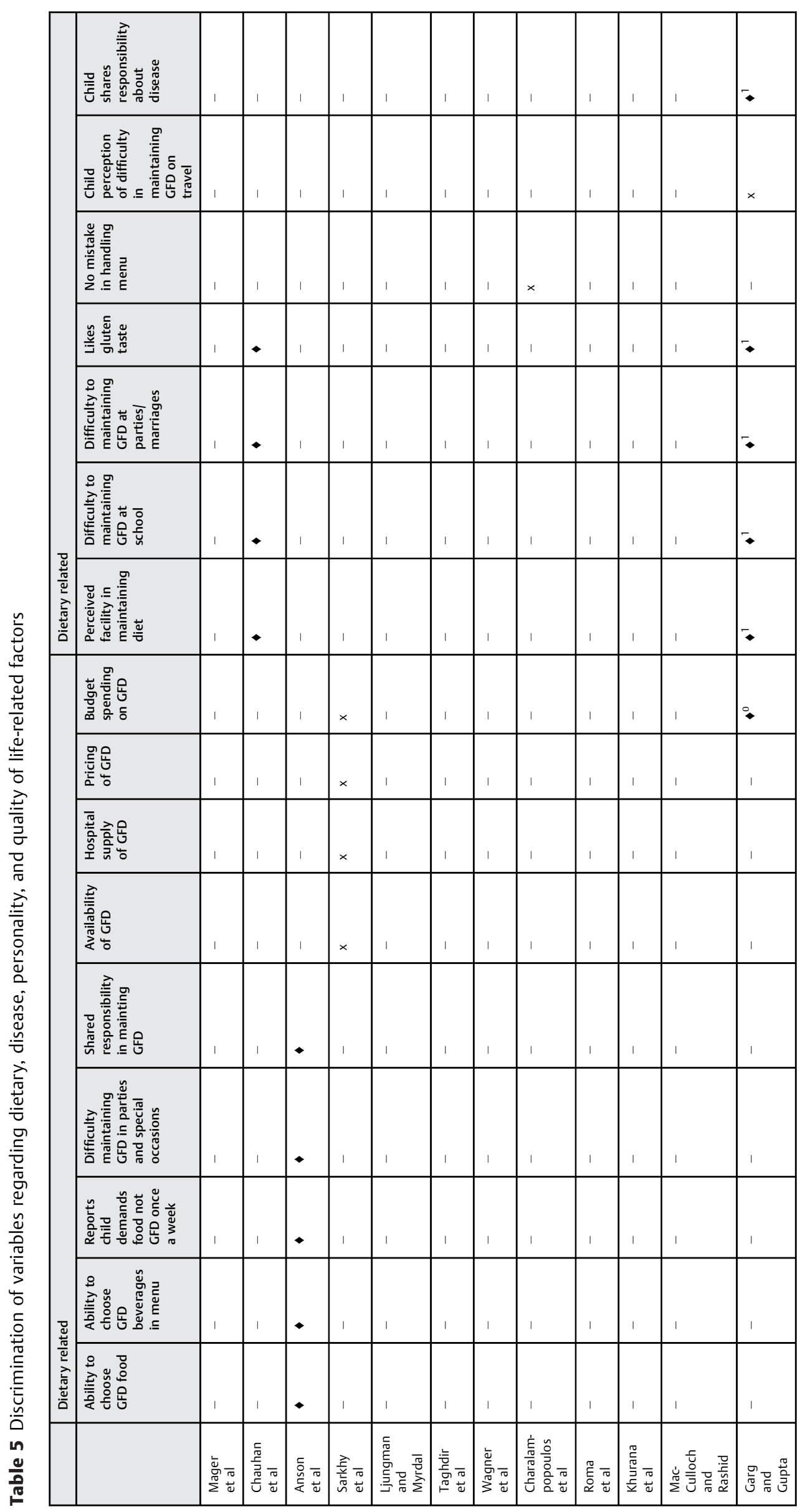




\begin{tabular}{|c|c|c|c|c|c|c|c|c|c|c|c|c|c|}
\hline & 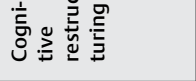 & 1 & 1 & 1 & 1 & 1 & 1 & $x$ & 1 & 1 & 1 & 1 & 1 \\
\hline 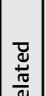 & 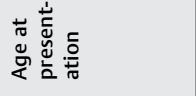 & 1 & 1 & 1 & 1 & 1 & 1 & 1 & 1 & 1 & 1 & & - \\
\hline 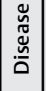 & 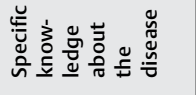 & , & , & $\times$ & 1 & 1 & 1 & 1 & 1 & 1 & 1 & 1 & \\
\hline & 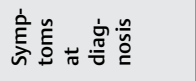 & , & । & 1 & 1 & 1 & 1 & 1 & 1 & 1 & 1 & I & $x$ \\
\hline & 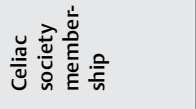 & 1 & 1 & 1 & 1 & 1 & 1 & 1 & 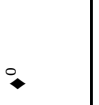 & - & 1 & 1 & \\
\hline & 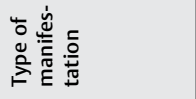 & 1 & 1 & 1 & 1 & 1 & $\mid 1$ & 1 & $x$ & 1 & 1 & I & 1 \\
\hline & 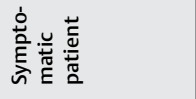 & 1 & 1 & 1 & 1 & 1 & 1 & 1 & $x$ & | & 1 & 1 & \\
\hline & 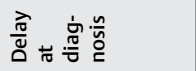 & , & , & । & । & 1 & 1 & 1 & $x$ & 1 & 1 & 1 & 1 \\
\hline & 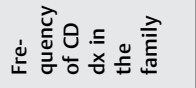 & , & 1 & 1 & 1 & 1 & $\mid$ & $x$ & 1 & 1 & 1 & 1 & \\
\hline & 离 & - & 1 & 1 & 1 & 1 & $\mid 1$ & 1 & 1 & 1 & 1 & 1 & 1 \\
\hline & 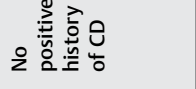 & - & 1 & 1 & 1 & 1 & $\mid 1$ & 1 & 1 & 1 & 1 & 1 & 1 \\
\hline & 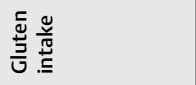 & $x$ & 1 & 1 & 1 & 1 & $\mid 1$ & 1 & 1 & 1 & 1 & 1 & 1 \\
\hline & 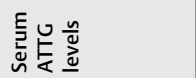 & $x$ & I & । & 1 & 1 & 1 & $x$ & 1 & 1 & 1 & 1 & 1 \\
\hline & 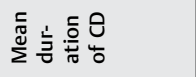 & , & । & । & 1 & 1 & $x$ & $x$ & 1 & 1 & 1 & 1 & 1 \\
\hline & 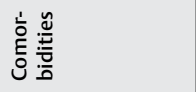 & 1 & 1 & 1 & 1 & 1 & $x$ & 1 & 1 & 1 & 1 & 1 & 1 \\
\hline & 总离 & , & , & , & • & 1 & 1 & 1 & 1 & 1 & 1 & $x$ & 1 \\
\hline & 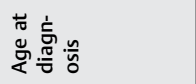 & I & 1 & 1 & • & 1 & 1 & $x$ & $x$ & 1 & 1 & 1 & $\times$ \\
\hline & 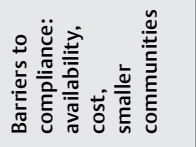 & I & 1 & 1 & 1 & 1 & 1 & 1 & 1 & | & 1 & I & 1 \\
\hline z & 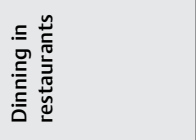 & 1 & 1 & 1 & 1 & 1 & 1 & 1 & 1 & 1 & 1 & - & 1 \\
\hline 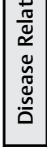 & 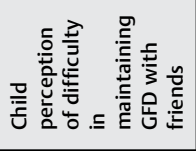 & I & I & 1 & 1 & 1 & । & 1 & I & I & 1 & 1 & 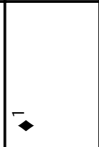 \\
\hline & & 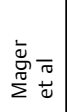 & 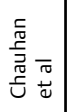 & 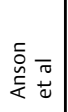 & 意 & 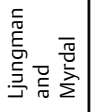 & 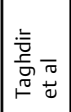 & 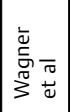 & 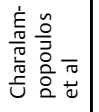 & 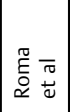 & 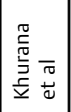 & 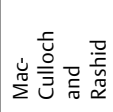 & 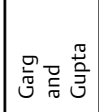 \\
\hline
\end{tabular}



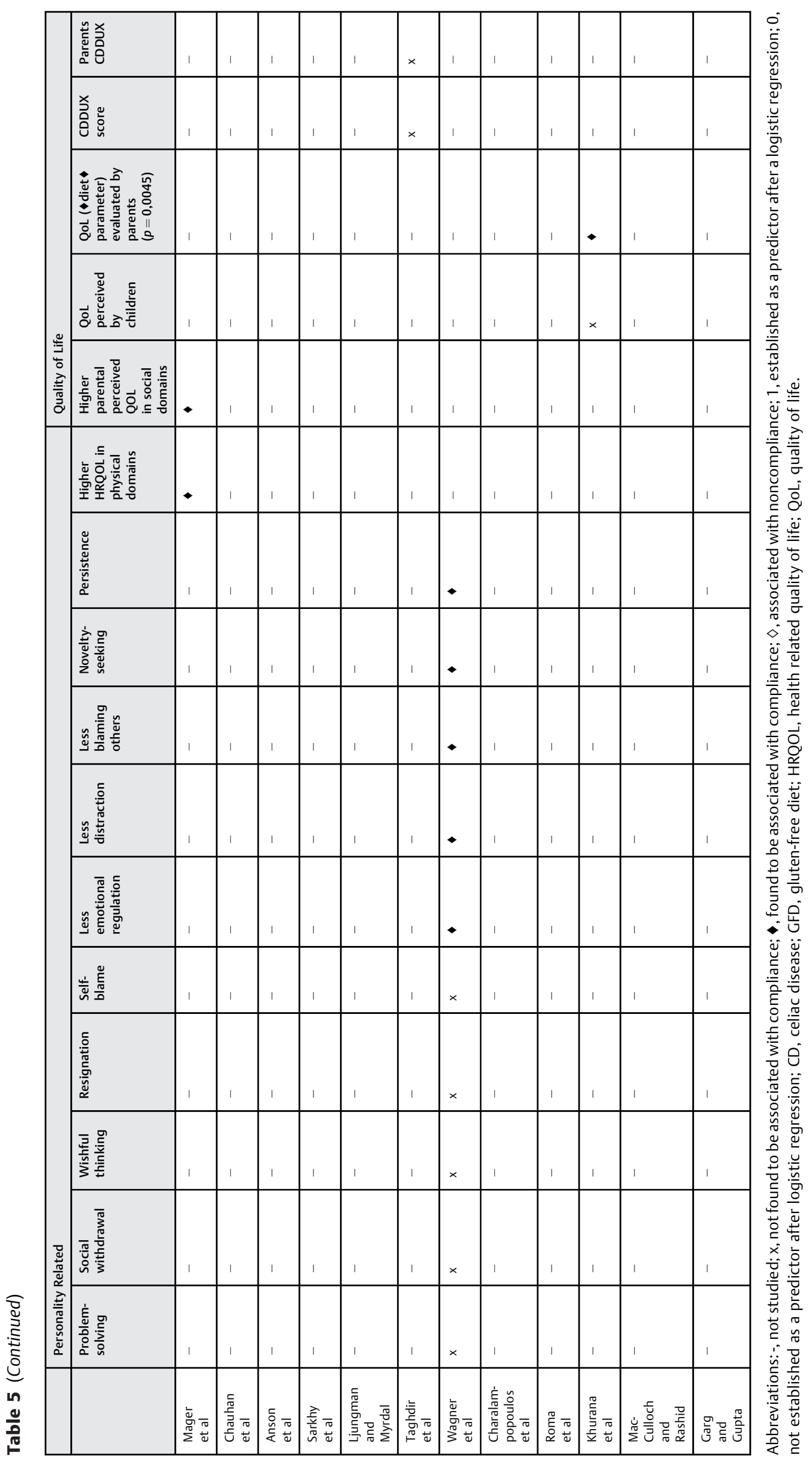
e10 Factors Affecting Compliance to a Gluten-Free Diet Macedo-Campos et al.

Table 6 Methods used to access compliance across the study

\begin{tabular}{|l|l|l|l|l|l|l|}
\hline Authors & Questionnaire & Biopsy & $\begin{array}{l}\text { Clinical } \\
\text { evaluation }\end{array}$ & $\begin{array}{l}\text { Anti-TG } \\
\text { antibodies }\end{array}$ & EMA & $\begin{array}{l}\text { Antireticulum } \\
\text { antibodies }\end{array}$ \\
\hline Mager et al & Applied & & & Applied & & \\
\hline Chauhan et al & Applied & & Applied & & & \\
\hline Anson et al & & Applied & Applied & & & Applied \\
\hline Sarkhy et al & Applied & & & & & \\
\hline Ljungman and Myrdal & Applied & & & & & \\
\hline Taghdir et al & Applied & & & Applied & Applied & \\
\hline Wagner et al & Applied & & & & & \\
\hline $\begin{array}{l}\text { Charalampopoulos } \\
\text { et al }\end{array}$ & Applied & & & Applied & Applied & \\
\hline Roma et al & Applied & & & Applied & Applied & \\
\hline Khurana et al & Applied & & & & & \\
\hline $\begin{array}{l}\text { MacCulloch } \\
\text { and Rashid }\end{array}$ & Applied & & & & & \\
\hline Garg and Gupta & Applied & & & & & \\
\hline
\end{tabular}

clinician interviews. ${ }^{11,17}$ Studies that used questionnaires had designed questionnaires specifically for the study or relied on previous validated questionnaires. Only one article used biopsies in follow-up. ${ }^{17}$ Despite doing so, the results were neither described nor used in conclusions. No article reported the use of urine gluten immunogenic proteins in monitoring disease activity. - Table 6 summarizes these findings.

\section{Age}

The works evaluating age treat this variable as categorical, dichotomous, and use different cut offs. Independently of the cut off value, these studies usually consider two groups, "younger" and "older" children.

In five papers (three of "Good" quality, ${ }^{11,13,18}$ one of "Medium" quality, ${ }^{14}$ and one of "Poor" quality ${ }^{10}$ ), younger age was significantly associated with compliance. In addition, two of these articles ${ }^{13,14}$ performed impact analysis, using logistic regression, and showed "older age" contributes to predict noncompliance. This observation was further supported by another two studies (one of "Good" quality ${ }^{15}$ and one of "Medium" quality ${ }^{12}$ ) showing older children to be significantly associated with noncompliance. In our sample, two papers (of "Medium" quality ${ }^{17}$ and of "Poor" quality ${ }^{8}$ ) were unable to report significant differences.

\section{Maternal Education}

In our review, eight articles evaluated maternal education. Four articles (two of "Good" quality ${ }^{11,13}$ and two of "Medium" ${ }^{5,17}$ quality) showed maternal education to be a significant factor for compliance to a GFD while another four (two of "Good" quality ${ }^{16,18}$ and two of "Poor" quality ${ }^{8,9}$ ) failed to show an effect.

Interestingly, one of the articles reporting an association between maternal education and compliance to a GFD, after performing a binary multivariate logistic regression analysis found this factor not to be a predictor of GFD compliance. ${ }^{13}$ In agreement, another article (“Good" quality ${ }^{11}$ ) showed a correlation between lower maternal education and noncompliance to GFD.

\section{Paternal Education}

Of the 12 articles included, six analyzed the influence paternal education has on a child's compliance. Of these, two articles (both of "Medium"5,17 quality) showed paternal education to be a significant factor for compliance to a GFD whereas another four (two of "Good" quality ${ }^{13,18}$ and two of "Poor" ${ }^{8,9}$ quality) failed to do so.

\section{Parental Knowledge of CD}

One article ("Good" quality ${ }^{11}$ ) demonstrated this parameter as positively influencing compliance to GFD while another ("Medium" quality ${ }^{17}$ ) found it not to influence it. Interestingly, one report ("Good" quality ${ }^{14}$ ) distinguishes parental knowledge in perceived and evaluated, showing that only the first was significantly associated with compliance to GFD. Moreover, after performing a multiple logistic regression they showed it is a predictor of compliance. At last, an article ("Good" quality ${ }^{13}$ ) reported statistical differences between the compliant and noncompliant groups, concerning parental knowledge of $C D$ but after conducting a binary multivariate logistic regression analysis, demonstrated this factor was not a predictor of compliance to GFD.

\section{Family Type}

Four articles evaluated "family type" as a dichotomic variable: a nuclear family-in which only the parents and their children live together, or a joint family-where the household inhabitants includes other family members. Two papers ("Good" quality ${ }^{11,13}$ ) reported that belonging to a nuclear family increases GFD compliance. In addition, one of the articles ${ }^{13}$ after performing a multivariable logistic regression analysis showed it to be a predictor of GFD compliance. The 
remaining two papers ("Medium"5 and "Poor"8 quality) showed no differences between compliant and noncompliant groups in relation to "family type."

\section{Child's Knowledge of CD}

The two works (of "Poor" quality ${ }^{9,10}$ ) that analyzed this variable showed a child's knowledge of CD as positively influencing compliance to GFD.

\section{Member of Celiac Society}

Two articles (one of "Medium"14 and one of "Poor"9 quality) evaluating the association between "adherence" and "celiac society membership" found significant differences between the compliant and noncompliant groups, with membership increasing GFD compliance. However, in one article, after performing a multivariate logistic regression analysis the effect was lost. ${ }^{14}$

\section{Quality of Life}

Three articles were found concerning the QoL. One article (of "Good" quality ${ }^{18}$ ) evaluated QoL on four different domains (social, emotional, school, and physical) and found that higher compliance was related with higher parental, perceived QoL, in social domains, and with child perceived QoL, in physical domains. Another article (of "Poor" quality ${ }^{8}$ ) evaluated QoL based on another scale-socio-demographic, QoL, diet, communication, and having CD. Using this scale, only the "diet" section as positively influenced compliance to GFD. Using the same scale, the last article (of "Medium" quality ${ }^{5}$ ) was unable to find any differences in the overall QoL.

\section{Maintaining GFD}

Three papers evaluated the difficulty in maintaining GFD. Two (of "Good"11,13 quality) stated children with higher compliance to the GFD considered gluten to have a "good taste" and found it less difficult to keep GFD generally at school and parties/marriages. Moreover, a binary multivariate analysis showed these factors were positive predictors of compliance. ${ }^{13}$ Another article (of "Medium" quality ${ }^{17}$ ) found that children who reported more difficulty in maintaining GFD were more frequent on the noncompliant group.

\section{Discussion}

Compliance within pediatric age is challenging, ${ }^{13}$ hence the importance of finding factors associated with compliance. Overall, our review showed three main factors associated with GFD compliance, "Family type" and "Parental knowledge of CD" positively increase GFD compliance while increasing "age" in pediatric population decreases compliance. In addition, maternal education and Celiac Society Membership are also related with GFD compliance.

In comparison to other topics regarding $C D$, little literature can be found regarding this subject, especially in pediatric populations. Our first literature search was unable to identify any systematic work on this matter and the first review published on the topic was on June 4, 2019 by Myléus et al. ${ }^{19}$ In this work, Myléus et al ${ }^{19}$ reviews the methods used to evaluate GFD compliance and associated risk factors while we propose to identify specific factors that positively or negatively affect GFD compliance.

In addition, we found the need to address compliance specifically in $C D$ patients without comorbidities since some of these, such as diabetes mellitus, imply additional lifestyle changes and diet restrains.

While it is logical to understand "age" as a having a major role in compliance, our results confirm this parameter is a good predictor of GFD compliance. As age increases so does autonomy in food selection. Furthermore, with age, there is also the need for social integration and peer approval. ${ }^{13}$ Also, considering an early age of diagnosis, it is less likely for a patient to develop unrestricted eating habits. All these are challenging when maintaining a different lifestyle, such as a GFD. Despite the absence of a cut-off value, there is a general consensus among the literature as per which changes in compliance are associated with age. ${ }^{10-15,18}$ Additionally, two articles demonstrated "older pediatric age" was associated with prediction of noncompliance. In fact, they stated that with each year of increase in age, the child had a $25^{13}$ and $15 \%$ ${ }^{14}$ less chance of remaining compliant to a GFD. Although one work reported opposite findings, its "Poor" quality limits its relevance to our analysis. ${ }^{8}$

When analyzing children's behavior toward maintaining a GFD, we expected to find differences between compliant and noncompliant children. Indeed, children that considered "gluten products as having better taste" displayed better GFD compliance and those reporting GFD "to be more difficult to maintain" showed less GFD compliance. ${ }^{11,13,17}$ While being quite challenging, as our data shows compliance decreases as children grow up, it is nonetheless a window of opportunity for pediatricians and dieticians to intervene by increasing the children's knowledge of CD and, consequently, GFD may become a more achievable goal. It should be noted that as the ability to "maintain a GFD" was self-reported, the relation of causality might be misleading so further investigation is required.

Concerning the effect of maternal education, we would expect this parameter to be a good predictor of GFD compliance due to the mother's role in food preparation and nutritional care. ${ }^{13}$ However, the results remain controversial as half of the studies found this parameter to be related to GFD compliance ${ }^{5,11,13,17}$ while the remaining did not, $8,9,16,18$ highlighting the need for further investigation.

To have a clearer picture on the influence of parental roles and since most works only study the maternal contribution, when available we also analyzed the influence of the father's education to GFD compliance. Two "Good" quality studies with large populations were unable to demonstrate differences in the impact of this parameter between complaint and noncompliant groups, ${ }^{13,18}$ although smaller studies of "Medium" quality supported this hypothesis. ${ }^{5,17}$ According to our results, it is likely this factor is not associated with GFD compliance, but it should be further explored in future works.

It is important to stress that there is no evidence of correlating parental knowledge of $\mathrm{CD}$ with formal education. Nonetheless, parental knowledge of $C D$ is related with compliance to GFD in pediatric populations, probably due to the major 
role that parents play in choosing their child's diet. In fact, Charalampopoulos et al showed parental knowledge is a predictor of compliance ${ }^{14}$ as children whose parents had a high perceived knowledge on $\mathrm{CD}$ were 3.3 times more likely to follow a strict diet. While this data was also supported by another "Good" quality work, ${ }^{11}$ two others ${ }^{13,17}$ of identical quality failed to identify it as a predictor of compliance emphasizing the need for further studies on this subject. Even though generalization is not possible at this time, physicians could still use parental knowledge of CD as a line of action to increase GFD compliance. This seems logical since parents with more knowledge will fail less in making diet choices, ${ }^{13}$ therefore their child complies more with a GFD.

Children feeding habits are the result of parental ability to drive their choices. Also, we report that decrease in compliance relates with older age. Therefore, it would be worth exploring this shift in their routine, as it comprises a change in compliance.

The analysis of "family" as a unit shows nuclear families are associated with compliance to GFD while joint families are related with noncompliance. ${ }^{5,8,11,13}$ One work demonstrated children from nuclear families to be four times more likely to maintain a $\mathrm{GFD}^{13}$-an effect thought to be associated with parents being more focused on their child's routine and environment. In a joint family, the amount of people eating on other diets may tempt the child to not comply..$^{13}$ Nevertheless, two articles were unable to find a relationship between GFD compliance with the family type. ${ }^{5,8}$ Given the lower quality of this work, there is a limitation to the relevance of these findings in our analysis. Importantly, these findings concerned a geographically limited region ${ }^{5,8,11,13}$ limiting its generalization due to potential sociodemographic differences.

One would expect children to be more knowledgeable of $C D$ to be more inclined to be GFD compliant given their raised awareness of the consequences of nonadherence and familiarity with gluten-free products. Indeed, two studies ${ }^{9,10}$ showed children's knowledge of CD to positively influence GFD compliance, however, due to the small population size evaluated, the overall quality of these reports is poor and the vague definition of "knowledge in CD,"10 extrapolation is limited.

Celiac Society Membership per se is positively related with compliance, ${ }^{9,14}$ which in light of our data are probably due to increased availability of information and contact with other $\mathrm{CD}$ patients. Importantly, the extent of its importance decreases with increasing levels of parental knowledge and with increased age of pediatric patients. It is important to note that only two studies demonstrated this factor as influencing compliance, ${ }^{9,14}$ one of them having "Poor" quality. ${ }^{9}$ Also, these results were based on specific populations ${ }^{9,14}$ (Greek), and therefore a selection bias may be present which partially limits its extrapolation to other populations.

High heterogenity is observed regarding QoL. This is due to the high variability of tools used to assess it and to the inconsistency of the studies overall quality. Therefore, is not possible to draw conclusions concerning this parameter.

Several factors limited our analysis. First, the heterogeneity of the tools used to measure GFD compliance leads to inconsistent results between reports. Standardization of research protocols would greatly enhance the quality and validity of future studies. Also, without having an objective measurement, such as t-TG levels, it is difficult to exclude contaminations.

Second, the definition of "GFD compliance" needs to be clarified. In the studies herein included, compliance was broadly defined as "a dichotomous variable in which a positive outcome is identifiable as the absence of awareness of gluten intake." By contrast, any awareness level of transgression to a GFD was considered as noncompliance. Our findings are corroborated by Myléus et $\mathrm{al}^{19}$ since they also could not find any method more reliable to assess compliance.

Third, most studies rely on parental information alone; which by itself is a potential source of bias since patients themselves do not report data. Of course, given the specific particularities of this population, it might not have been possible to obtain the data otherwise.

Fourthly, an additional source of bias was associated with the lack of standardization in the age categories at study. The lower and higher limits of each age group varied greatly between reports, compromising its evaluation. Finally, there was an overall lack of quality in what concerns study design and the presentation of results.

As to the reports' quality assessment and subsequent extrapolation of results, we would like to note that since the majority of the studies were cross-sectional, observational by nature, the results obtained could not be standardized as predictors, as the relationship between exposure and outcome cannot be fully discriminated. For this same reason, in the quality assessment, most studies consistently received a negative score for the study design. Additionally, we stress the need for further studies that establish a correlation between exposure and outcome.

\section{Conclusion}

Undoubtedly, GFD is strongly related with social and environmental contexts. The interconnection between parental features and children outcomes became clearer. Despite some of them being unchangeable, such as age or family type, with this work, we were able to show evidence that modifiable factors, parental knowledge on CD per example, can also play major roles in compliance. Nevertheless, the causality between these factors and compliance still remains unclear. Therefore, the need for further knowledge in causality relations is in order so that compliance rates, among pediatric populations, can be increased.

Regarding this, age was proved to influence compliance as young children consistently display better compliance rates. More so, children of informed parents and a nuclear family household were independently positive influencers of compliance. All these predictors should be taken into account in clinical practice when evaluating CD patients.

Lastly, it is important to state that a systematic approach to compliance should be established, only then GFD compliance will be fully understood. 


\section{Authors' Contributions}

V.M.-C. and R.M.-C. conceptualized the work, collected, analyzed, and interpreted, drew and reviewed the first draft and ensured for the integrity and accuracy in the work. H.A. conceptualized the work, reviewed the first draft, and ensured for the integrity and accuracy in the work. F.P.-R. reviewed the first draft and ensured for the integrity and accuracy in the work. All the authors approved the final manuscript as submitted and agree to be accountable for all aspects of the work.

These authors contributed equally to this work.

\section{Funding}

This work was funded by the Portuguese Science Foundation Project PTDC/SAUNEU/108557/2008, COMPETE and FEDER.

\section{Conflict of Interest}

None declared.

\section{References}

1 Mustalahti K, Catassi C, Reunanen A, et al; Coeliac EU Cluster, Project Epidemiology. The prevalence of celiac disease in Europe: results of a centralized, international mass screening project. Ann Med 2010;42(08):587-595

2 Lundin KEA, Qiao S-W, Snir O, Sollid LM. Coeliac disease-from genetic and immunological studies to clinical applications. Scand J Gastroenterol 2015;50(06):708-717

3 Lindfors K, Ciacci C, Kurppa K, et al. Coeliac disease. Nat Rev Dis Primers 2019;5(01):3

4 Nardecchia S, Auricchio R, Discepolo V, Troncone R. Extra-intestinal manifestations of coeliac disease in children: clinical features and mechanisms. Front Pediatr 2019;7(March):56

5 Taghdir M, Honar N, Mazloomi SM, Sepandi M, Ashourpour M, Salehi M. Dietary compliance in Iranian children and adolescents with celiac disease. J Multidiscip Healthc 2016;9:365-370

6 Study Quality Assessment Tools | National Heart, Lung, and Blood Institute (NHLBI). Available at: https://www.nhlbi.nih.gov/healthtopics/study-quality-assessment-tools. Accessed June 4, 2019
7 Moher D, Liberati A, Tetzlaff J, Altman DGPRISMA Group. Preferred reporting items for systematic reviews and meta-analyses: the PRISMA statement. PLoS Med 2009;6(07):e1000097

8 Khurana B, Lomash A, Khalil S, Bhattacharya M, Rajeshwari K, Kapoor S. Evaluation of the impact of celiac disease and its dietary manipulation on children and their caregivers. Indian J Gastroenterol 2015;34(02):112-116

9 Roma E, Roubani A, Kolia E, Panayiotou J, Zellos A, Syriopoulou VP. Dietary compliance and life style of children with coeliac disease. J Hum Nutr Diet 2010;23(02):176-182

10 Ljungman G, Myrdal U. Compliance in teenagers with coeliac disease-a Swedish follow-up study. Acta Paediatr 1993;82(03): 235-238

11 Chauhan JC, Kumar P, Dutta AK, Basu S, Kumar A. Assessment of dietary compliance to gluten free diet and psychosocial problems in Indian children with celiac disease. Indian J Pediatr 2010;77 (06):649-654

12 Wagner G, Zeiler M, Grylli V, et al. Coeliac disease in adolescence: coping strategies and personality factors affecting compliance with gluten-free diet. Appetite 2016;101:55-61

13 Garg A, Gupta R. Predictors of compliance to gluten-free diet in children with celiac disease. Int Sch Res Notices 2014;2014:248402

14 Charalampopoulos D, Panayiotou J, Chouliaras G, Zellos A, Kyritsi E, Roma E. Determinants of adherence to gluten-free diet in Greek children with coeliac disease: a cross-sectional study. Eur J Clin Nutr 2013;67(06):615-619

15 MacCulloch K, Rashid M. Factors affecting adherence to a glutenfree diet in children with celiac disease. Paediatr Child Health 2014;19(06):305-309

16 Sarkhy AA, El Mouzan MI, Saeed E, et al. Clinical characteristics of celiac disease and dietary adherence to gluten-free diet among Saudi Children. Pediatr Gastroenterol Hepatol Nutr 2015;18(01):23-29

17 Anson O, Weizman Z, Zeevi N. Celiac Disease: Parental Knowledge and Attitudes of Dietary Compliance. Vol. 85. 1990. Available at: www.aappublications.org/news

18 Mager DR, Marcon M, Brill H, et al. Adherence to the gluten-free diet and health-related quality of life in an ethnically diverse pediatric population with celiac disease. J Pediatr Gastroenterol Nutr 2018;66(06):941-948

19 Myléus A, Reilly NR, Green PHR. Rate, risk factors and outcomes of non-adherence in pediatric patients with celiac disease: a systematic review. Clin Gastroenterol Hepatol 2020;18(03): 562-573 